\title{
Código Ingenios y el sistema de patentes: ¿una propuesta innovadora o la receta hacia un es- tancamiento tecnológico?!
}

\author{
Sophia Espinosa Coloma, J.S.D \\ Universidad San Francisco de Quito
}

\begin{abstract}
Resumen
El Código Orgánico de Economía Social del Conocimiento e Innovación (Código Ingenios) es un nuevo proyecto que se discute en la Asamblea ecuatoriana que tiene como fin democratizar el conocimiento, concibiendo a éste como un bien de interés público. El Código Ingenios en los elementos de fondo del sistema de propiedad intelectual -inclusive los derechos de patentes- es consistente con lo establecido por el Acuerdo sobre los aspectos de los Derechos de Propiedad Intelectual relacionados con el Comercio (ADPIC). No obstante, introduce cambios sustantivos en lo referente al material patentable, las exclusiones de patentabilidad y las excepciones de patentabilidad. Estas modificaciones no solo pueden ser consideradas contrarias al ADPIC, sino que podrían ir en contra de la innovación, generando un estancamiento tecnológico en el país y disuadiendo las inversiones en investigación y desarrollo.
\end{abstract}

\begin{abstract}
Currently Ecuador's Congress is working in a new intellectual property legal regime called Código Ingenios. Its main objective is the dissemination of knowledge, as it is considered a good of public interest. Código Ingenios is consistent with the Agreement on Trade-Related Aspects of Intellectual Property Rights (TRIPS) in most main aspects, including the patent law regime. Nevertheless, it incorporates material modifications to the patentable subject matter, the exclusions from patentability and the exceptions to IP rights that are not only conflicting with the TRIPS. Agreement, but also reduces incentives to innovation, potentially causing a technological stagnation and deterring investments in research and development.
\end{abstract}

Palabras clave: Código Orgánico de Economía Social del Conocimiento e Innovación / Código Ingenios / Patentes; Materia Patentable / Patentes de Genes; Exclusiones de Patentabilidad / Conocimiento Tradicional / Excepciones de Patentabilidad / ADPIC / TRIPS.

Key words: Código Orgánico de Economía Social del Conocimiento e Innovación / Cógido Ingenios / Patent Law / Patent subject matter / Gene patents / Exclusion of 
patentability / TRIPS flexibilities / Traditional Knowledge / Patent Exceptions / TRIPS Agreement.

Sumario: 1. Introducción; 2. Código Ingenios y Derecho de Patentes: ¿Promoción de la Innovación o aislamiento tecnológico?; 2.1 Materia Patentable; 2.2 Exclusiones de Patentabilidad; 2.3 Excepciones del Derecho de Patentes; 3. Conclusiones

\section{Introducción}

En una conversación sobre el derecho de patentes, Fritz Machlup concluyó que "if we did not have a patent system, it would be irresponsible on the basis of our present knowledge of its economic consequences, to recommend instituting one. But since we have had a patent system for a long time, it would be irresponsible, on the basis of our present knowledge, to recommend abolishing it". ${ }^{2}$ El sistema de patentes existente no es perfecto, trae consigo algunos efectos, no todos positivos como es el caso de los costos sociales. Sin embargo, es un sistema que ha permitido sentar estándares mínimos para la protección de las creaciones del intelecto humano, generando así seguridad jurídica y facilitando el comercio y la transferencia de tecnología entre naciones.

El sistema de propiedad intelectual de forma general, y el de patentes de forma específica, son concebidos como herramientas para la promoción de la innovación y el progreso. De esta manera, el derecho de patentes consiste en el otorgamiento de derechos de exclusiva sobre la invención, a favor del inventor, con el fin de que reciba una recompensa e incentivo por su trabajo. A cambio de esta protección, el inventor se compromete a divulgarla, con el objetivo de que esta información contribuya al desarrollo tecnológico y a la innovación en general, beneficiando así a la sociedad. Actualmente, existe una preocupación mundial sobre la efectividad del presente sistema de propiedad intelectual contenido en el Acuerdo sobre los aspectos de los Derechos de Propiedad Intelectual relacionados con el Comercio (ADPIC) ${ }^{3}$, por lo que se ha considerado la necesidad de dinamizar el sistema, con el fin de que se constituya en un latente motor de la innovación y creación en todas las esferas de la propiedad intelectual. ${ }^{4}$ El Ecuador, dentro de esta nueva tendencia, ha desarrollado el Código Orgánico de Economía Social del Conocimiento e Innovación (Código Ingenios), así su objeto es "generar un marco legal en el que se estructure la economía social de los conocimientos, la creatividad y la innovación". ${ }^{5}$

\footnotetext{
2 Kitch, Edmund, "Patents", The New Palgrave Dictionary of Economics and the Law 4, in The Economics of Intellectual Property 4, Volume II, Peter Newman (ed.), 1998.

3Acuerdo sobre los aspectos de los Derechos de Propiedad Intelectual relacionados con el Comercio, disponible en https:// www.wto.org/spanish/docs_s/legal_s/27-trips_02_s.htm

4 International Conference on Intellectual Property and Development, Ginebra 7-8 Abril 2016.

5 Código Orgánico de Economía Social del Conocimiento e Innovación, Art.1 disponible en http://coesc.educacionsuperior.
} 
El Código Ingenios concibe al conocimiento como un bien de interés público, teniendo como base la democratización del mismo. Establece así dentro de sus principios que "el conocimiento constituye un bien de interés público, su acceso será libre y no tendrá más restricciones que las establecidas en este Código, la Constitución, los tratados e instrumentos internacionales y la Ley; y, su distribución se realizará de manera justa, equitativa y democrática". Con estas premisas el Código Ingenios, en el área de patentes, se ciñe básicamente a lo establecido en el ADPIC. No obstante, introduce modificaciones sustantivas puntuales.

Por tanto, este estudio tiene como objetivo determinar si el Código Ingenios, en el área de patentes, representa una propuesta viable, consistente con la naturaleza de estos derechos y con la promoción de la investigación y desarrollo, o por el contrario, son propuestas que podrían acarrear un impacto negativo en la innovación y producción tecnológica.

\section{Código Ingenios y Derecho de Patentes: ¿Promoción de la Innovación o aisla- miento tecnológico?}

El tema del Derecho de Patentes se encuentra regulado por los ADPIC, Tratado que establece un set de estándares mínimos orientados a reducir las distorsiones e impedimentos en el mercado internacional y para promover una protección adecuada de los derechos de propiedad intelectual entre los países miembros de la Organización Mundial de Comercio (OMC). Uno de los objetivos de los ADPIC es asegurar que las disposiciones relativas a la aplicación de los derechos de propiedad intelectual no constituyan en sí mismos obstáculos o barreras al comercio legítimo. ${ }^{6}$

La Decisión de la Comunidad Andina, al igual que la Ley de Propiedad Intelectual actual siguen los parámetros establecidos en los ADPIC. Por su parte, el Código Ingenios, si bien no realiza cambios significativos de fondo, introduce modificaciones de carácter sustantivo, que no solo podrían considerarse inconsistentes con el ADPIC, sino generar un impacto negativo en la innovación.

gob.ec/index.php/C\%C3\%B3digo_Org\%C3\%A1nico_de_Econom\%C3\%ADa_Social_del_Conocimiento_e Innovaci\%C3\%B3n1998.

6Acuerdo sobre los aspectos de los Derechos de Propiedad Intelectual relacionados con el Comercio, Preámbulo, párr.1, disponible en: https://www.wto.org/spanish/docs_s/legal_s/27-trips_02_s.htm 


\subsection{Materia Patentable}

En el área de derecho de patentes, el Artículo 27 del ADPIC ${ }^{7}$ es importante, en razón de que éste cubre la materia patentable, los mínimos estándares de protección y las excepciones de patentabilidad determinadas en consideración a los intereses públicos.

El Artículo 27.1 señala que las patentes deben otorgarse cuando concurran las siguientes circunstancias:

[... las patentes podrán obtenerse por todas las invenciones, sean de productos o de procedimientos, en todos los campos de la tecnología, siempre que sean nuevas, entrañen una actividad inventiva y sean susceptibles de aplicación industrial (...) las patentes se podrán obtener y los derechos de patente se podrán gozar sin discriminación por el lugar de la invención, el campo de la tecnología o el hecho de que los productos sean importados o producidos en el país. ${ }^{8}$

Esta provisión extiende la materia protegible, de manera que cualquier producto o proceso puede ser protegido bajo el derecho de patentes si cumple los requisitos de patentabilidad -novedad, nivel inventivo, aplicación industrial. La razón fundamental para extender la materia protegible, obedece a las múltiples y controversiales exclusiones de patentabilidad que algunos países tenían en áreas clave como farmacéutica, químicos y las industrias de alimentos. ${ }^{9}$ Para el tiempo en que se realizaba la Ronda de Uruguay, alrededor de 50 países no otorgaban derechos de

7 Ibídem, Art. 27 Materia patentable:1. Sin perjuicio de lo dispuesto en los párrafos 2 y 3, las patentes podrán obtenerse por todas las invenciones, sean de productos o de procedimientos, en todos los campos de la tecnología, siempre que sean nuevas, entrañen una actividad inventiva y sean susceptibles de aplicación industrial.(5) Sin perjuicio de lo dispuesto en el párrafo 4 del artículo 65, en el párrafo 8 del artículo 70 y en el párrafo 3 del presente artículo, las patentes se podrán obtener y los derechos de patente se podrán gozar sin discriminación por el lugar de la invención, el campo de la tecnología o el hecho de que los productos sean importados o producidos en el país. 2. Los Miembros podrán excluir de la patentabilidad las invenciones cuya explotación comercial en su territorio deba impedirse necesariamente para proteger el orden público o la moralidad, inclusive para proteger la salud o la vida de las personas o de los animales o para preservar los vegetales, o para evitar daños graves al medio ambiente, siempre que esa exclusión no se haga meramente porque la explotación esté prohibida por su legislación. 3 . Los Miembros podrán excluir asimismo de la patentabilidad: a) los métodos de diagnóstico, terapéuticos y quirúrgicos para el tratamiento de personas o animales; b) las plantas y los animales excepto los microorganismos, y los procedimientos esencialmente biológicos para la producción de plantas o animales, que no sean procedimientos no biológicos o microbiológicos. Sin embargo, los Miembros otorgarán protección a todas las obtenciones vegetales mediante patentes, mediante un sistema eficaz sui generis o mediante una combinación de aquéllas y éste. Las disposiciones del presente apartado serán objeto de examen cuatro años después de la entrada en vigor del Acuerdo sobre la OMC.

8 Ibídem, Art. 27.1.

9 Véase Correa, Carlos, Trade Related Aspects of Intellectual Property Rights, Oxford University Press, Great Britain, 2007, p. 71. Véase también de Carvalho, Nuno Pires The TRIPS Regime of Patent Rights 141, Kluwer Law ed., London, 2003. 
patentes a invenciones en el área de medicina, alimentos y bebidas. ${ }^{10}$ Por tanto, se trataba de definir un sistema que evite exclusión de patentabilidad en campos que requieren protección, por el alto riesgo existente a casusa de la significativa cantidad de recursos, trabajo e inversión realizada.

La posibilidad de obtener patentes para cualquier invención, sean productos o procesos en cualquier campo de la tecnología, abrió el campo para que se obtengan patentes de productos en áreas como medicina, farmacéutica, biotecnología, agroquímica, entre otros. El reconocimiento de la protección a través del derecho de patentes en estas áreas, tenía como objetivo principal el asegurar la continuación y fortalecimiento de la investigación y desarrollo de éstas. Como resultado se puede resaltar que la protección bajo el derecho de patentes fue concebida como un mecanismo para promover el desarrollo de nuevos productos, a cambio de derechos de propiedad intelectual sobre los resultados de esta investigación y desarrollo. ${ }^{11}$

En este sentido, el Código Ingenios en su Art. 253 adopta el estándar establecido por el ADPIC, extendiendo la materia patentable a todo producto o procedimiento, en todo campo de la tecnología, siempre que cumpla con los requisitos de patentabilidad. Por otro lado, Art. 252 establece que el “[...] sistema de patentes constituye una herramienta para promover el desarrollo industrial y tecnológico y para la consecución del buen vivir". ${ }^{12}$ Por tanto, podemos establecer que en parte el Código Ingenios es consistente con el ADPIC, en razón de que concibe al derecho de patentes como un mecanismo para fomentar la innovación y por ende el desarrollo tecnológico e industrial. Sin embargo, omite la importancia de la utilización del derecho de patentes como un medio para incentivar al inventor y fomentar el comercio internacional. Una de las bases del sistema de propiedad intelectual es la justa retribución al inventor con el fin de estimular la innovación, para lograr nuevos productos que mejoren los estándares de vida de la sociedad.

Asimismo, uno de los fines del ADPIC es precisamente lograr una armonización de la normativa internacional de patentes, que provea con seguridad jurídica a los desarrolladores y usuarios de tecnología en todos los campos, con el fin de fomentar el comercio. Si no se concibe al sistema de propiedad intelectual en función de todas sus dimensiones y actores, se tendrá una normativa sesgada y orientada a satisfacer las necesidades, únicamente, de una de las aristas del sistema.

\footnotetext{
10 Ibídem

11 Véase Correa, Carlos, Trade Related Aspects, p. 275.

12 Código Orgánico de Economía Social del Conocimiento e Innovación, Art.252 disponible en http://coesc.educacionsuperior. gob.ec/index.php/C\%C3\%B3digo_Org\%C3\%A1nico_de_Econom\%C3\%ADa_Social_del_Conocimiento_e_ Innovaci\%C3\%B3n.
} 


\subsubsection{Genes: ¿Materia Protegible por el Derecho de Patentes?}

El Art. 27.1 del ADPIC establece que las patentes estarán disponibles para cualquier invención. Sin embargo, el término invención no ha sido definido por el ADPIC. Esto se da para que cada uno de los países miembros tenga la discrecionalidad de adoptar su propia definición de invención en sus legislaciones domésticas. ${ }^{13}$ Este punto tiene un impacto significativo dentro del campo de los recursos genéticos, puesto que cada país decidirá si el material genético, genes, secuencias de ADN, entre otros podrán o no ser materia patentable. "This manner of regulating protection may well entail consequences in connection with the patenting of natural materials as well, in particular biological material of natural origin (e.g. DNA, cell lines, etc), for the TRIPs Agreement does not contain any mandatory rules on the essential delimitation between 'discoveries' and 'inventions'". ${ }^{14}$

Consecuentemente, cada país miembro de la OMC tiene la facultad de adoptar la posición más conveniente en función de sus realidades o necesidades. Así, los países miembros pueden optar por la visión estricta de invención o definir una posición más amplia e incluir algunos tipos de "descubrimientos" que pueden reunir los requisitos de patentabilidad dadas las circunstancias. De esta manera, con el fin de clarificar estas dos posturas en el área de recursos genéticos el Nuffield Council on Bioethics ha establecido que:

$[\ldots]$ genes are naturally occurring entities that are there to be discovered, like new species or new planets. They are not invented. In our common usage of the term, a "discovery" is the acquisition of knowledge of a new but already existing fact about the world. An "invention", on the other hand, is something that someone creates or develops which did not previously exist. Thus, on the usual interpretation of the words, it seems apparent that the identification of a gene is a discovery, since genes exist in the world, in our bodies..$^{15}$

Sin embargo, ¿qué pasa cuando los genes son objeto de la intervención humana? ¿Qué grado de intervención del intelecto humano debe haber para considerarlos materia patentable o, simplemente, bajo ninguna circunstancia podrían patentarse? La respuesta no es fácil y dependerá de la corriente adoptada por cada legisla-

13 Straus, J. "Implications of the TRIPs Agreement in the field of patent law", From Gatt to TRIPs- The Agreement on Trade-Related Aspects of Intellectual Property Rights F-K Beier and G Schricker (eds.), Max Planck Institute for Foreign and International Patent, Copyright and Competition Law, Munich, 1996, p.187.

14 Ibídem

15 Nuffield Council on Bioethics, The ethics of patenting DNA. A discussion paper 23, London, 2002. 
ción. Por ejemplo, Estados Unidos de Norteamérica y la Unión Europea otorgan patentes a genes que cumplen los requisitos de patentabilidad. ${ }^{16}$ Estados Unidos sigue el estándar de patentabilidad establecido en el caso Diamond v. Chakrabarty (1980), en este caso la Corte Suprema de Estados Unidos estableció que microorganismos vivos desarrollados por el ser humano, constituyen materia patentable bajo la Sección 101 de la US Patent Act. ${ }^{17}$ Así, la Corte establece que una patente puede ser obtenida sobre "anything under the sun that is made by man". ${ }^{18}$

Este estándar hizo que, por algunos años, se permita la patentabilidad de material genético aislado y purificado. Sin embargo, en junio de 2013 la Corte Suprema de Estados Unidos, en el caso de Myriad Genetics ${ }^{19}$, establece un nuevo parámetro para la evaluación del cumplimiento de los requisitos de patentabilidad: novedad, utilidad y no obviedad. La nueva interpretación realizada por la Corte Suprema deja claro que los genes, secuencias genéticas y material genético no son materia patentable por el simple hecho de ser aislados, por cuanto no se ha producido una alteración a la información contenida por estos genes, estando ésta en el mismo estado que provee la naturaleza. La sentencia dada en este caso no prohíbe la patentabilidad de genes en términos absolutos, solo de aquellos que se encuentran en estado natural, aun cuando hayan sido sometidos a un proceso de purificación y/o aislamiento. De esta manera, el ADN complementario que cumple los requisitos de patentabilidad satisfactoria y suficiente puede ser objeto de patentabilidad.

cDNA does not present the same obstacles to patentability as naturally occurring, isolated DNA segments. As already explained, creation of a cDNA sequence from mRNA results in an exons-only molecule that is not naturally occurring. Petitioners concede that cDNA differs from natural DNA in that "the non-coding regions have been removed". Brief for Petitioners 49. They nevertheless argue that cDNA is not patent eligible because "[t]he nucleotide sequence of cDNA is dictated by nature, not by the lab technician". Id., at 51. That may be so, but the lab technician unquestionably creates something new when cDNA is made. cDNA retains the naturally occurring exons of DNA, but it is distinct from the DNA from which it was derived. As a result, cDNA is not a "product of nature" and is patent eligible under $\S 101$, except insofar as very short series of DNA may have no intervening

\footnotetext{
16 Véase Correa, Carlos, Trade Related Aspects, p. 272.

17 United States Supreme Court. Diamond v. Chakrabarty, 447 U.S. 303, 1980.

18 Ibídem, p.309.

19 United States Supreme Court. Association for Molecular Pathology et al., Petitioners v .Myriad Genetics, Inc., et al., No. 12-398, 13 de junio de 2013. 2013 WL 2631062.
} 
introns to remove when creating cDNA. In that situation, a short strand of cDNA may be indistinguishable from natural DNA. ${ }^{20}$

Por otro lado, en el caso de la Unión Europea, que en su Directiva 98/44 relativa a la protección jurídica de las invenciones tecnológicas, en su Art. 3.2 establece como materia patentable a "la materia biológica aislada de su entorno natural o producida por medio de un procedimiento técnico podrá ser objeto de una invención, aun cuando ya exista anteriormente en estado natural". ${ }^{21}$ Asimismo, el Art. 5.2 determina que: "Un elemento aislado del cuerpo humano u obtenido de otro modo mediante un procedimiento técnico, incluida la secuencia o la secuencia parcial de un gen, podrá considerarse como una invención patentable, aun en el caso de que la estructura de dicho elemento sea idéntica a la de un elemento natural" ${ }^{22}$

En el caso ecuatoriano, la actual Ley de Propiedad Intelectual sigue la línea del ADPIC, por tanto, no se regula nada referente a la patentabilidad del material genético, constituyéndose una línea gris en este campo. No obstante, el Código Ingenios adopta la visión más estricta, prohibiendo de forma explícita la patentabilidad de genes y material genético en general. De esta manera, en el Art. 254 numeral 2 se establece que: "No se consideran invenciones: 2. El todo o parte de seres vivos tal como se encuentran en la naturaleza, los procesos biológicos naturales, el material biológico existente en la naturaleza, o aquel que pueda ser aislado, inclusive genes, proteínas, genoma o germoplasma de cualquier ser vivo". ${ }^{23}$ Así, al no considerarse invención, el material genético aislado y purificado no es materia protegible. Sin embargo, ¿qué pasa con el ADN complementario? ¿Es patentable bajo la propuesta del Código Ingenios? Es importante señalar, que ya que se decidió regular de manera explícita lo referente a la patentabilidad de material genético, se debe dejar en claro si podría considerarse materia protegible las invenciones basadas en material genético que ha sido modificado de forma significativa por el ser humano y reúne los requisitos de patentabilidad.

La patentabilidad de genes tiene dos posturas. Por un lado, la de aquellos que apoyan la patentabilidad de material genético con el fin de incentivar a los inventores. Esto se da porque el derecho de patentes otorga a su titular un derecho de exclusi-

\footnotetext{
20 Ibídem p.10.

21 Directiva 98/44/CE del Parlamento Europeo y del Consejo relativa a la protección jurídica de las invenciones biotecnológicas. Artículo 3.2 de 6 de julio de 1998.

22 Ibídem, Art. 5.2.

23 Código Orgánico de Economía Social del Conocimiento e Innovación, Art. 254 disponible en http://coesc.educacionsuperior. gob.ec/index.php/C\%C3\%B3digo_Org\%C3\%A1nico_de_Econom\%C3\%ADa_Social_del_Conocimiento_e_ Innovaci\%C3\%B3n
} 
vidad, que le permite excluir a terceros del uso y aprovechamiento de la invención protegida. De igual manera, esta posición, es defendida por aquellos que promueven la teoría de la tragedia de los comunes, que plantea que el otorgamiento de los derechos de patentes sobre genes evitaría una sobreexplotación de los mismos. "El proceso de concesión de patentes en las secuencias de ADN se ha sustentado en lo que se denomina Tragedy of Commons. Mediante esta teoría se explica por qué un sistema en el que los recursos son compartidos por quienes estén interesados en ellos, lleva a la sobreexplotación de los recursos y a la falta de innovación (Heller y Eisenberg, 1998)". 24

Por otro lado, la postura que va en contra de la patentabilidad de genes, fundamenta su posición en el hecho de que los genes son materia existente en la naturaleza, por tanto, un gen aislado y purificado no puede considerarse una invención por no haber una intervención o aporte significativo del ser humano. De esta manera, se considera que el otorgamiento de patentes sobre genes constituye una política que va en contra de la invención, por cuanto los inventores tendrán que pedir un sinnúmero de permisos a los titulares de los derechos de patentes, con el fin de acceder al material genético para la investigación. De esta manera, se trata de evitar la generación de patent thickets que se constituyan en obstáculos para la innovación tecnológica. A esta postura se la conoce también como la teoría del anticommons.

An anticommons is "a property regime in which multiple owners hold effective rights of exclusion in a scarce resource". Such scenarios, moreover, often result in underdevelopment of that resource. [...] Applied to biomedical research, the tragedy of the anticommons explains how patents may actually deter innovation. While earlier commentators focused on innovation as a unitary act by a single player, expositors of the tragedy of the anticommons recognize the multiparty, multiphase mechanics of contemporary research and development. Patents on upstream research tools can inhibit downstream research that depends upon those tools as critical inputs to experimentation. Thus, for example, patents on research tools necessary to screen therapeutic agents can inhibit a pharmaceutical company's efforts to develop marketable drugs. In short, "intellectual property rights have threatened to restrict access to materials and techniques that are critical for future research". While patents have contributed to unprecedented levels of investment in biomedical research and development, in the long run their exclusionary function may hinder rather than accelerate biomedical research. ${ }^{25}$

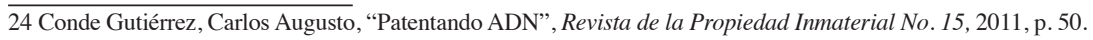


Por tanto, en este punto se puede establecer que la postura adoptada por el Código Ingenios con respecto a la no patentabilidad de genes aislados y purificados no afecta a la innovación tecnológica. De tal manera, es una posición que se ajusta a las realidades de país y al estado de la industria biotecnológica. Asimismo, la no patentabilidad de genes en el caso ecuatoriano tiene por objeto promover la innovación, permitiendo el acceso de los investigadores al material genético base. Ahora bien, sería interesante que se deje en claro qué pasa con el material genético que además de haber sido purificado y aislado ha sido sometido a un proceso innovador. El cumplimiento de los requisitos de patentabilidad son suficientes? ¿Cuál es el estándar a seguir con respecto al requisito de nivel inventivo? Es decir, el nivel inventivo debe ser suficiente y ¿qué se entiende por suficiente es esta área? La posibilidad de que no se permita patentar invenciones consistentes en modificaciones a material genético que satisfacen plenamente los requisitos de patentabilidad, no solo que iría en contra de lo establecido en el ADPIC, sino que impactaría de forma negativa a la innovación tecnológica en el país.

\subsection{Exclusiones de la Patentabilidad}

Como se ha establecido anteriormente, el ADPIC establece los estándares mínimos de protección de los derechos de propiedad intelectual. Sin embargo, el tratado también establece flexibilidades a lo largo del texto, con el fin de facilitar a los países miembros la adopción de la normativa de propiedad intelectual en función a sus realidades nacionales. Dentro de las flexibilidades del ADPIC se encuentran las exclusiones de patentabilidad, contenidas en los artículos 27.2 y $27.3 .^{26}$

De esta manera, la actual Ley de Propiedad Intelectual es un ejemplo de las flexibilidades del ADPIC, ya que se acogen todas las excepciones de patentabilidad en el texto. El Código Ingenios, siguiendo esta línea, adopta las mismas excepciones

\footnotetext{
Law Journal Company, 2004, pp.673-674.

26 Acuerdo sobre los aspectos de los Derechos de Propiedad Intelectual relacionados con el Comercio, Art. 27.(2)(3) disponible en https://www.wto.org/spanish/docs_s/legal_s/27-trips_02_s.htm Los Miembros podrán excluir de la patentabilidad las invenciones cuya explotación comercial en su territorio deba impedirse necesariamente para proteger el orden público o la moralidad, inclusive para proteger la salud o la vida de las personas o de los animales o para preservar los vegetales, o para evitar daños graves al medio ambiente, siempre que esa exclusión no se haga meramente porque la explotación esté prohibida por su legislación.
}

3. Los Miembros podrán excluir asimismo de la patentabilidad:

a) los métodos de diagnóstico, terapéuticos y quirúrgicos para el tratamiento de personas o animales; b) las plantas y los animales excepto los microorganismos, y los procedimientos esencialmente biológicos para la producción de plantas o animales, que no sean procedimientos no biológicos o microbiológicos. Sin embargo, los Miembros otorgarán protección a todas las obtenciones vegetales mediante patentes, mediante un sistema eficaz sui generis o mediante una combinación de aquéllas y éste. Las disposiciones del presente apartado serán objeto de examen cuatro años después de la entrada en vigor del Acuerdo sobre la OMC. 
de patentabilidad propuestas por el ADPIC. No obstante, la extiende a los conocimientos tradicionales. Así el Art. 259 establece que:

Artículo 259.- Invenciones no patentables.- No será patentable lo siguiente:

1. Las invenciones cuya explotación comercial deba impedirse necesariamente para proteger el orden público o la moralidad, inclusive para proteger la salud o la vida de las personas o de los animales o para preservar los vegetales o para evitar daños graves al medio ambiente o ecosistema. A estos efectos, no se considerará contraria al orden público o a la moral la explotación comercial de una invención, por la sola existencia de una disposición legal o administrativa que prohíba o que regule dicha explotación;

2. Los métodos de diagnóstico, terapéuticos y quirúrgicos para el tratamiento de personas o animales;

3. Las plantas y los animales, así como los procedimientos esencialmente biológicos para obtención de plantas o animales que no sean procedimientos no-biológicos o microbiológicos; y,

4. Los conocimientos tradicionales. ${ }^{27}$

Este enfoque va de la mano con lo establecido en el artículo 322 de la Constitución, que prohíbe todo tipo de apropiación de conocimientos colectivos ${ }^{28}$, así como del Art.402 que prohíbe la obtención de derechos de propiedad intelectual sobre productos derivados y sintetizados obtenidos a partir del conocimiento tradicional. ${ }^{29} \mathrm{Al}$ hablar de la protección y gestión del conocimiento tradicional, se puede identificar dos posiciones, la protección defensiva y la positiva. La protección defensiva se orienta al desarrollo de cualquier mecanismo para evitar la apropiación de conocimiento tradicional por parte de terceros no autorizados. ${ }^{30}$ La protección defensiva encuentra su objetivo primordial en el derecho de patentes, así con el objetivo de evitar la adquisición de derecho de patentes sobre el conocimiento tradicional por parte de terceros. La Constitución del Ecuador y el Código Ingenios siguen este enfoque, pues al evitar

\footnotetext{
27 Código Orgánico de Economía Social del Conocimiento e Innovación, Art. 259 disponible enhttp://coesc.educacionsuperior. gob.ec/index.php/C\%C3\%B3digo_Org\%C3\%A1nico_de_Econom\%C3\%ADa_Social_del_Conocimiento_e_ Innovaci\%C3\%B3n

28 Constitución de la República del Ecuador. Artículo 322. Registro Oficial No.449 de 20 de octubre de 2008.

29 Ibidem., Art.402

${ }_{30}$ Véase Pires de Carvalho, Nuno, From the Shaman's Hut to the Patent Office: A Road Under Construction, in Biodiversity and the Law 247, Charles McManis ed., Earthscan, USA, 2007. Véase también WIPO, WIPO, Intellectual Property and Traditional Knowledge 6, WIPO Publication No.920 (E), disponible en: http://www.wipo.int/edocs/pubdocs/en/tk/920/wipo_pub_920.pdf
} 
la obtención del derecho de patentes, y en general de todo tipo de propiedad sobre el conocimiento tradicional, lo que verdaderamente busca es evitar que terceros se conviertan en los titulares del conocimiento comunitario.

Defensive protection aims at ensuring that existing TK is not patented by third parties - ideally, by ensuring that relevant TK is taken fully into account when a patent is examined for its novelty and inventiveness. Normally, a claimed invention in a patent application is assessed against the so- called "prior art" - the defined body of knowledge that is considered relevant to the validity of a patent. For example, if TK has been published in a journal before the applicable date of a patent application, it is part of the relevant prior art, and the application cannot validly claim that TK as an invention - the invention would not be considered novel. In recent years, concern has been expressed that TK should be given greater attention as relevant prior art, so that patents are less likely to cover existing publicly disclosed TK. ${ }^{31}$

Por otro lado, la protección positiva está orientada a la creación de un sistema que otorga derechos sobre el conocimiento tradicional a sus tenedores, de esta manera, los empodera para que gestionen este conocimiento y para tomar acción en contra de un mal uso o de una explotación no autorizada del conocimiento tradicional. ${ }^{32}$ Por tanto, la protección positiva utiliza mecanismos legales para asegurar la protección del conocimiento tradicional.

The options for positive protection include existing IP laws and legal systems (including the law of unfair competition), extended or adapted IP rights specifically focused on TK (sui generis aspects of IP laws), and new, stand-alone sui generis systems which give rights in TK as such. Other nonIP options can form part of the overall menu, including trade practices and labeling laws, the law of civil liability, the use of contracts, customary and indigenous laws and protocols, regulation of access to genetic resources and associated TK, and remedies based on such torts as unjust enrichment, rights of publicity, and blasphemy. ${ }^{33}$

Como se ha establecido, la protección positiva contempla los derechos de propiedad existentes para la protección efectiva del conocimiento tradicional. Dentro del debate sobre la protección del conocimiento tradicional, hay aún escepticismo

\footnotetext{
31 WIPO, WIPO, Intellectual Property, p.26. 
sobre la efectividad de utilizar los derechos de propiedad existentes, por considerar que la naturaleza del conocimiento tradicional hace que estos derechos sean inconsistentes e incluso contradictorios con la materia de protección. ${ }^{34}$ Una de las principales razones para fundamentar este escepticismo está basada en los orígenes de los conocimientos tradicionales y de los derechos de propiedad intelectual, y los intereses subyacentes de estos.

In the growing international debate over the legal protection of traditional knowledge, one frequently hears the view expressed that the traditional knowledge of indigenous peoples and local communities does not readily fit into the existing intellectual property regimes of the industrialized world, that these regimes promote the interests of the industrialized world and encourage what has come to be called (and vilified as) "biopiracy". ${ }^{35}$

No obstante, la experiencia ha demostrado lo contrario, probando que el sistema de propiedad intelectual existente puede proteger los derechos tradicionales de manera eficaz, legal y legítima. De acuerdo con la Organización Mundial de Propiedad Intelectual, "existing IP laws have been successfully used to protect against some forms of misuse and misappropriation of TK, including through the laws of patents, trademarks, geographical indications, industrial designs, and trade secrets" ${ }^{36}$

Consideramos que con el fin de promover la innovación el Código Ingenios no debería excluir la posibilidad de obtener derechos de patentes sobre conocimientos tradicionales, pues es una medida que no solo constituye un obstáculo para la innovación, sino que va en contra de los intereses de los tenedores de conocimientos tradicionales, entorpeciendo la repartición de beneficios establecida en la Convención sobre Diversidad Biológica.

\subsubsection{Conocimiento Tradicional y Derecho de Patentes}

Muchos sectores consideran que el derecho de patentes no es aplicable al conocimiento tradicional, dadas las características y orígenes propios de éste. Sin embargo, podemos establecer que en ciertas circunstancias el conocimiento tradicional que cumpla con los requisitos de patentabilidad (novedad, aplicabilidad industrial y nivel inventivo), puede ser objeto de protección. Esta posibilidad ha sido sugerida

\footnotetext{
34 Véase McManis, Charles, "Fitting Traditional Knowledge Protection and Biopiracy Claims into the Existing Intellectual Property and Unfair Competition Framework", Intellectual Property and Biological Resources, (Burton Ong ed., Marshall Cavendish, Singapore, 2004, 430-31.

35 Ibídem, p. 425.

36 WIPO, WIPO, Intellectual Property, p. 17.
} 
en particular para la protección del conocimiento tradicional medicinal. ${ }^{37}$ De esta manera, "[...] when practitioners innovate within the traditional framework, they have been able to use the patent system to protect their innovations. For example, in 2001 China granted 3300 patents for innovations within the field of Traditional Chinese Medicine [... $]^{\prime 38}$

$\mathrm{Al}$ analizar la posibilidad de proteger al conocimiento tradicional bajo el derecho de patentes, nos encontramos con dos posibles impedimentos de fondo, el cumplimiento del requisito de novedad y la concepción del inventor como un individuo. A continuación se verá como bajo ciertas circunstancias estos obstáculos se pueden sobrellevar, permitiendo así la aplicación del derecho de patentes.

El conocimiento tradicional es concebido como una "creation over a long period of time in which it has been passed down from generation to generation". ${ }^{39}$ Este carácter colectivo del conocimiento tradicional genera problemas con el requisito de novedad. ${ }^{40}$ De conformidad con la visión occidental, el conocimiento que pasa de generación en generación es parte del dominio público. ${ }^{41}$ Por tanto, ¿cómo el conocimiento tradicional puede cumplir con el requisito de novedad si es parte del dominio público? Esta aseveración contiene una idea equivocada, por cuanto no todo el conocimiento tradicional es compartido con toda la comunidad. En efecto, el conocimiento tradicional más relevante es reservado por pocos o un solo miembro de la comunidad, como es el caso del Shaman. "Recent investigations, [...], have shown that not all indigenous knowledge is communally shared, and not all of it is considered to be in the public domain. Various healing methods have been reported to have been held under a secrecy regime". 42 Asimismo, los tenedores del conocimiento tradicional han desarrollado sus propios mecanismos para regular el acceso y uso del conocimiento tradicional, por tanto mal se podría decir que es parte del dominio público. Al respecto Dutfield establece que:

Many traditional societies have their own custom-based "intellectual property" systems, which are sometimes quite complex. Customary rules governing access to and use of knowledge do not necessarily differ all that

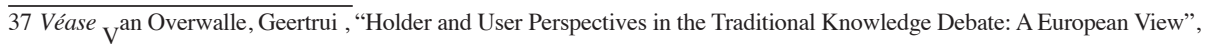
Biodiversity and the Law, Charles McManis ed., 2007.

38 WIPO, WIPO, Intellectual Property, p. 18.

39 Lewis, Walter H.; Ramani, Veena, Ethics and Practice in Ethnobiology: Analysis of the International Cooperative Biodiversity Group Project in Peru, in Biodiversity and the Law, Charles McManis ed., Earthscan, USA, 2007, p. 394.

40 Von Lewinski, Silke; Von Hahn, Anja Indigenous Heritage and Intellectual Property 65, (Silke von Lewinski \& Anja von Hahn eds., Kluwer Law International, The Netherlands, 2004.

41 Long, Doris Traditional Knowledge and the Fight for the Public Domain, 5j Marshall Rev.Intell.Prop.1. 2006, pp. 307-321. 42 Van Overwalle, Geertrui, "Holder and User", p. 359.
} 
widely from western intellectual property formulations, but in the vast majority of cases they almost certainly do. Nonetheless, there is a tendency to treat such rules with disrespect or to ignore them as if they do not exist. However, knowledge thought to be part of the public domain may in some cases turn out under customary law to remain subject to the legal claims of individuals and communities. Even if one disregards customary law, the unauthorized dissemination or use of certain publicly available traditional knowledge could sometimes be challenged on the basis of concepts existing in the western legal system, such as copyright, breach of confidence and misappropriation. Accordingly and in consequence, nothing is being taken from the public domain that should be there, but only what should not be. [...] The public domain is being promoted in opposition to privatization as part of a debate about intellectual property rights, a discussion that does not easily accommodate the specific interests and claims of non-Western societies. Why is this the case? Disclosed TK has from the distant past to the present been treated as belonging to nobody. Consequently, many indigenous peoples' representatives are concerned that pro-public domain rhetoric, sympathetic as many of them are about the sentiments behind it, may inadvertently threaten their rights. Indeed, the public domain concept is problematic from the perspective of many traditional societies in which TK holders or others, such as tribal elders, have permanent responsibilities concerning the use of such knowledge, irrespective of whether it is secret, is known to just a few people, or is known to thousands of people throughout the world. Custodianship responsibilities do not necessarily cease to exist just because the knowledge has been placed in the so-called public domain. There is no doubt that a tremendous amount of TK has been disclosed and disseminated over the years without the authorization of the holders. ${ }^{43}$

Por otro lado, se analizará la concepción del inventor como individuo. En este punto se tiene que considerar que no todo el conocimiento tradicional es creado por toda la comunidad, de esta manera no se descarta la existencia de un conocimiento tradicional desarrollado por un individuo. "In general, creation of TK is an incremental and collective process, but it does not follow that TK is not the products of individuals. Depending on the customary laws and principles applicable to particular situations, nothing stands in the way of recognizing an individual creation as a

43 Dutfield, Graham, Protecting Traditional Knowledge: Pathways to the Future 8-9, Graham Dutfield ed., International Centre for Trade and Sustainable Development (ICTSD), Switzerland, 2006. 
genuine piece of TK" ${ }^{44}$ Asimismo, estos tipos de conocimiento tradicional no solo pueden ser protegidos bajo el derecho de patentes, sino incluso por el derecho de autor. Al respecto, Balick establece que "one way of strengthening the position of the traditional healer employed in the Belize project has been to consider these people as colleagues and teachers, rather than as informants. [...] By including traditional healers who provided information for research as co-authors or providing acknowledgment using their names, all parties benefit". ${ }^{45}$

Otra solución para este problema es el uso del concepto de coinventor. Para utilizar este concepto es necesario que "each of the joint inventors have contributed to the inventive conception, working on the same subject matter and making the same contributions to the inventive thought ant to the final result" ${ }^{46} \mathrm{~S}$. Biber-Klemm al hablar sobre el caso Americano establece:

In the USA, for instance, the statute recognizes the notion of joint inventorship. ${ }^{47}$ The main requirements imposed for joint inventorship are that each of the joint inventors must contribute to the inventive element of the protected invention and that they must collaborate with each other. More specifically, the joint inventors must produce an invention by their aggregate efforts and must work on the same subject matter. ${ }^{48}$ Further, while it may be difficult to distinguish prior art from the distinct contributions to a single invention, the statute recognizes that joint inventors do not have to work together at the same time. It is sufficient that the contribution of the joint inventor that works first in time constitutes an integral part of the process of joint invention. ${ }^{49}$ Finally, the joint inventors do not need to collaborate on each of the claims made in a patent application and do not need to have made the same type of contribution or contributed equally to the invention. ${ }^{5051}$

Consecuentemente, si el tenedor de conocimiento tradicional contribuye de forma directa y consciente para el desarrollo de una invención, puede ser considerado

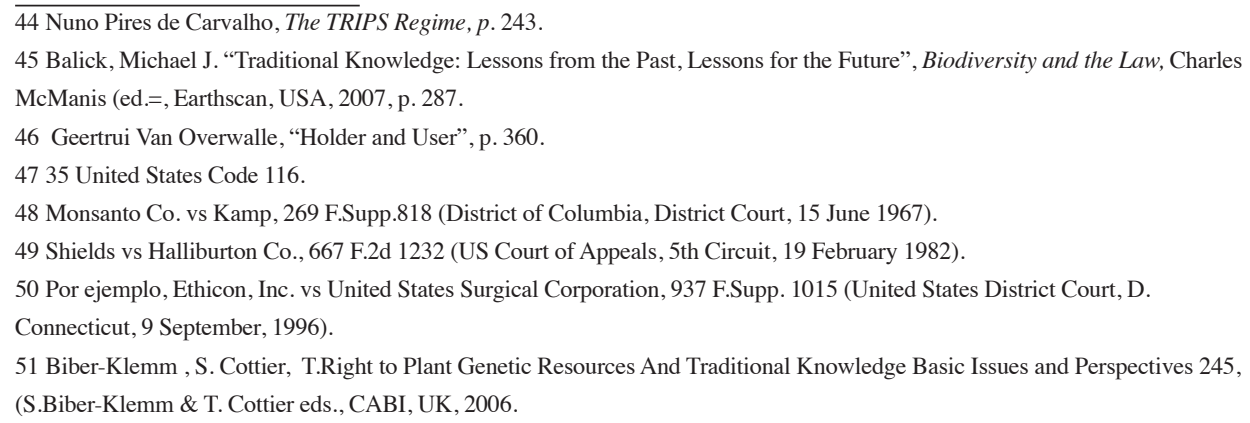


como coinventor. En otras palabras, si se reúnen los requisitos y su contribución es relevante a la invención final, entonces el tenedor de conocimiento tradicional puede ser considerado coinventor.

The question of inventorship and entitlement to apply for a patent may be at issue in cases where the inventive concept has been derived in whole or in part from a TK holder, such as an individual TK practitioner, but that person is not recognized as the or $\mathrm{a}[\mathrm{n}]$ inventor of the invention as claimed. This question therefore have potential relevance to traditional knowledge systems, and their potential relevance to patents for inventions that are TK, that use TK or that are otherwise based on TK. Entitlement to apply for a patent, inventorship, rights to ownership, obligations arising from noninventive contributions, enforcement of contractual obligations, and the formal recognition of ownership, licensing and security interests, are all legally significant issues in acquiring, holding and enforcing patent rights, [and] - and thus may play a role in access and benefit-sharing. ${ }^{52}$

Por otro lado, el hecho de que el tenedor del conocimiento tradicional no pueda calificar como coinventor, no debe ser una razón para privarlo por los beneficios obtenidos a raíz de la invención. Una posible solución es la utilización de la figura de la copropiedad a través de la cesión de derechos de patentes a favor de los tenedores de conocimiento tradicional. Esto se puede evidenciar en el Proyecto International Cooperative Biodiversity Group (ICBG) en Perú. El ICBG es un proyecto que apoya la investigación y desarrollo de medicinas, a través de productos biológicos, así "discovery and development of pharmaceutical and other useful agents from natural products can promote economic opportunities and enhanced research capacity in developing countries while conserving the biological resources from which these products are derived". ${ }^{53}$ El ICBG es considerado como una de las buenas prácticas en la protección de conocimientos tradicionales, ya que incorpora varios mecanismos para conseguir este fin y una justa repartición de beneficios con sus tenedores. ${ }^{54}$

Each agreement recognizes that the traditional knowledge of the indigenous people is their cultural legacy and that the people have a right for such knowledge to be protected from the public domain. They state that such knowledge is being provided voluntarily and is being retained in con-

\footnotetext{
52 WIPO/GRTKF/IC/13/7, of September 18, 2008- "Recognition of Traditional Knowledge within the Patent System", paragraph 41 .

53 Lewis, Walter H.; Ramani, Veena, Ethics and Practice, p. 400.

54 Ibídem, pp. 400-410.
} 
fidence. Should such information prove valuable, then the original IPR of the indigenous people over such knowledge would be preserved through the filing of appropriate patents, and by the inventors assigning shared ownership of the patents to the indigenous federations. The agreements also recognize the ownership and patrimony of the Peruvian state over certain tangible resources (whole plants) collected by the researchers in Peru for scientific purposes and for making extracts, fractions and isolating compounds of potential commercial use as new pharmaceuticals [... The project incorporated the goals of the CBD by accessing genetic material and traditional knowledge with the PIC of the people involved. It recognized the rights of the indigenous people to their traditional knowledge and compensated them fairly. It also recognized the sovereign rights of the government of Peru, provided them with benefits, particularly by involving source country institutions and transferring back knowledge and technology to the country. ${ }^{55}$

Por tanto, el momento en que se decide eliminar la posibilidad de obtener derechos de patentes sobre el conocimiento tradicional y los productos obtenidos con base en el conocimiento tradicional, se está atentando a los intereses de las comunidades nativas. De esta manera, se elimina una herramienta que constituye un mecanismo viable para garantizar la repartición justa de beneficios. Así también, se elimina el incentivo hacia los inventores, quienes ante la imposibilidad de obtener una patente, posiblemente identificarán comunidades en países vecinos, en donde puedan realizar su trabajo y a la vez obtener una protección para su invención, de forma que todos los involucrados puedan ser partícipes de los beneficios de la investigación. Consideramos que el Ecuador, al ser un país megadiverso, debería enfocar sus esfuerzos en la creación de un sistema de protección positiva de conocimiento tradicional, de forma que sus tenedores se vean empoderados y a la vez motivados para convertirse en titulares de derechos sobre el conocimiento tradicional, que les permita no solo proteger sus conocimientos, sino constituirse en pieza clave para la innovación.

\subsection{Excepciones del Derecho de Patentes}

Considerando que ningún derecho es absoluto, el ADPIC contempla excepciones de los derechos de propiedad intelectual, permitiendo un uso no regulado y libre acceso a las obras o innovaciones protegidas. Estas excepciones se encuentran comprendidas dentro de las flexibilidades del ADPIC, de manera que se permite que los 
Estados regulen las excepciones de conformidad con sus necesidades. Así, el Art. 30 del ADPIC establece que "los Miembros podrán prever excepciones limitadas de los derechos exclusivos conferidos por una patente, a condición de que tales excepciones no atenten de manera injustificable contra la explotación normal de la patente ni causen un perjuicio injustificado a los legítimos intereses del titular de la patente, teniendo en cuenta los intereses legítimos de terceros". ${ }^{56}$ Por tanto, las excepciones deben ser manejadas dentro de un enfoque razonable, que vaya de la mano con las necesidades sociales, pero también considerándose como medidas extraordinarias, por cuanto podrían menoscabar los derechos de sus titulares y afectar a la innovación, investigación y desarrollo.

En este sentido el Código Ingenios incluye una preocupante clasificación que impacta directamente al derecho de patentes. De esta manera, el Art. 86 introduce una tipología de bienes que establece que “(p) los efectos del presente Código, en función de los derechos de propiedad intelectual que les son aplicables, se establece la siguiente tipología de bienes: 1. Bienes que garantizan derechos fundamentales; [ ... ]". ${ }^{57}$ Así el Código Ingenios establece que

Artículo 87.- Bienes que garantizan derechos fundamentales.- Los bienes que garantizan derechos fundamentales y que se encuentren protegidos por derechos de propiedad intelectual, son de interés público y gozarán de un tipo de protección que permita satisfacer las necesidades básicas de la sociedad, para lo cual, sin perjuicio del uso de limitaciones y excepciones a los derechos, se permitirán otros usos sin autorización del titular de conformidad con lo establecido en este Código y en los tratados internacionales de los que Ecuador es parte.

Lo dispuesto en el párrafo precedente será aplicable también para los derechos que recaigan sobre la información no divulgada y los datos de prueba, sobre productos farmacéuticos y químico- agrícolas. ${ }^{58}$

Estas normas si bien buscan una propiedad intelectual más humana y que fomente la plena vigencia de los derechos fundamentales, dejan de lado la realidad y los intereses de los titulares de derechos de propiedad intelectual en general y de derechos de patentes en particular. De esta manera, la normativa en lugar de crear con-

\footnotetext{
56 Acuerdo sobre los aspectos de los Derechos de Propiedad Intelectual relacionados con el Comercio, Art.30 disponible enhttps://www.wto.org/spanish/docs_s/legal_s/27-trips_02_s.htm

57 Código Orgánico de Economía Social del Conocimiento e Innovación, Art.86 disponible enhttp://coesc.educacionsuperior. gob.ec/index.php/C\%C3\%B3digo_Org\%C3\%A1nico_de_Econom\%C3\%ADa_Social_del_Conocimiento_e Innovaci\%C3\%B3n 58 Ibídem
} 
diciones para que los inventores dinamicen sus procesos de investigación y desarrollo, incorpora nuevas excepciones, que van más allá de las licencias obligatorias, y que no solo menoscaban los derechos de los titulares de propiedad intelectual, sino que desincentivan la innovación.

Uno de los sectores clave que se ve afectado por esta norma es el de la industria farmacéutica. El acceso a medicamentos es una necesidad y clamor mundial. Sin embargo, para lograrlo de manera efectiva, los Estados deben crear las condiciones socio económicas, políticas y jurídicas para que la industria farmacéutica logre reducir el riesgo, los costos y así pueda proporcionar medicamentos al alcance de todos los sectores de la población. En este sentido, el derecho de patentes sobre medicamentos no debe ser visto como un obstáculo para su acceso, sino como una herramienta que viabiliza la generación de estas drogas con fines terapéuticos. La idea de establecer un sistema orientado a las necesidades sociales, permitiendo otros usos no autorizados más allá de los establecidos en la normativa interna e internacional, con el fin de sociabilizar el acceso a estos productos, responde a la visión de que las patentes constituyen monopolios, cuyo efecto es el incremento de los costos, creando así barreras para el acceso y disfrute de las innovaciones.

\subsubsection{Patentes, Costo Social y Monopolio}

El derecho de patentes otorga derechos de exclusiva a favor del inventor por el desarrollo de una invención- sea ésta un producto o proceso- que ofrece una nueva solución técnica a un problema específico. Por tanto, en este sentido la patente sí implica el otorgamiento de un monopolio a favor del titular del derecho de patentes. No obstante, en la mayoría de casos este monopolio es solo literal mas no de mercado, en el sentido de que muchos productos patentados tienen competidores y por ende productos sustitutos. Edmund Kitch establece que "the description of a patent as a monopoly depends in part on the ambiguity of the term monopoly. A patent is certainly a monopoly in the sense that any property right is a monopoly- it is the exclusive right to control the use of the subject matter of the patent claim". ${ }^{59}$

Si se asume la posición de que los derechos de patentes generan monopolios, a causa de los derechos de exclusiva otorgados a favor del titular, se puede determinar que algunos de los efectos se resumen en los costos sociales. En este sentido se puede identificar dos costos sociales significativos: En primer lugar, el excedente del consumidor se reduce, ya que los consumidores tienen que pagar más para utilizar la invención, que según la teoría de los bienes públicos debería ser de libre acceso.

59 Kitch, Edmund, "Patents", The New Palgrave Dictionary of Economics and the Law 4, The Economics of Intellectual Property 4, Volume II, Peter Newman (ed.), 1998. 
En segundo lugar, algunos consumidores dejen de usar el producto, por lo que sufren una pérdida, porque ya no están disfrutando de las ventajas de esta invención.

When market price rises above the competitive level, consumers who continue to purchase the sellers' product at the new, higher price suffer loss exactly offset by the additional revenue that the sellers obtain at the higher price. Those who stop buying the product suffer a loss not offset by any gain to the sellers. This is the deadweight loss from supracompetitive pricing and in traditional analysis its only social costs. ${ }^{60}$

Esta posición asume un escenario extremo, en donde toda patente genera un monopolio, causando costos sociales significativos. Sin embargo, si se elimina el sistema de patentes, desaparecerían los costos sociales? En este punto es importante considerar que una de las bases del sistema de patentes y de propiedad intelectual, es la posibilidad de proveer con incentivos a los inventores para que continúen investigando y desarrollando nuevas invenciones. De ahí que, es altamente probable que sin la protección otorgada por el derecho de patentes, la actividad inventiva se vea menoscabada, o que los inventores busquen nuevos mecanismos de protección como el secreto, impidiendo la divulgación del conocimiento que prevé el sistema de patentes. Por tanto, qué costo social es mayor- aquel generado por el Sistema de patentes o aquel que simplemente imposibilita a la sociedad a tener acceso a nuevas invenciones, porque simplemente no existen?

Consecuentemente, es importante generar mecanismos efectivos para simplificar la transferencia del conocimiento, porque cualquier herramienta que retrasa el acceso al conocimiento trae como consecuencia un costo social. Sin el sistema de patentes o dentro de un sistema que reconozca el derecho de patentes, pero que prevea excepciones relevantes a éste, nos enfrentamos a tres escenarios. Primero, que el inventor deje de inventar. Segundo, que el inventor decida esconder sus innovaciones. Tercero, que el inventor busque medidas contractuales con rigurosos candados, para asegurar la transacción. Como resultado, sin un sistema efectivo de patentes es probable que la sociedad se vea privada del desarrollo tecnológico. Esta posición es compartida por Posner, quien señala que:

The patent laws embody a somewhat similar economizing technique. In their absence inventors would expend substantial resources on preserving the secrecy of their innovations. Their efforts in this direction would generate indirect as well as direct social costs, by retarding the spread of knowledge. By

60 Richard A., Posner, "The Social Cost of Monopoly and Regulation", , he New Palgrave Dictionary of Economics and the Law 4, The Economics of Intellectual Property 4, Volume II, Peter Newman (ed.), 1998. 
providing a legal remedy against stealing inventions, the patent laws reduce the level of such expenditures in much the same way as the existence of legal penalties for theft reduces the level of resources that people devote to protecting their property from thieves. ${ }^{61}$

Por otro lado, como señalamos anteriormente, no todas las patentes causan un monopolio de mercado. Así, Kitch establece que:

A patent is not usually a monopoly in the sense of price theory- an exclusive right to sell into a market with a downward sloping demand curve. The invention which is the subject of a patent claim is not the same as a product market. Many patents in fact turn out to be worthless because the product or process covered by their claims cannot be profitable exploited in competition with other available alternatives. ${ }^{62}$

De ahí que algunos expertos en el área hayan concluido que el derecho de patentes y la normativa de competencia, son cuerpos legales complementarios. Gallini y Trebilcock desarrollan esta teoría de la siguiente manera:

Intellectual property embodies information that is a public good: an inventor's consumption of the information does not preclude others from consuming it and so, in the absence of property rights, an innovation will be imitated. In recognition of the public good nature of IP, patent law provides intellectual property rights (IPRs) in innovations; without IPRs, the incentive to invest in innovation would be diminished. The law also recognizes that the dynamic benefits from IPRs come at an allocative cost, in that the use of the innovation will be suboptimal: information is relatively costless to transmit, and its efficient price is therefore zero; exclusionary rights, on the other hand, enable the patentee to set a positive price for the information, thus reducing the output and flow of that information. By giving rights to an innovation that are exclusive but limited in scope and duration, an IPR awarded under patent law attempts to strike the appropriate balance between these competing concerns. Competition law impacts on the exercise of the innovator's rights-and therefore on its reward-by restricting certain practices involving the IPR. [ . . ] IP grant seeks to protect property rights, and, in so doing, limits competition. In contrast, competition law generally reflects the premise that consumer welfare is best served by removing impediments to competition. $[\ldots]$ There is, therefore, a growing willingness to restrict competition today

61 Ibídem, p.21.

62. Kitch, Edmund, "Patents", p. 4. 
in order to promote competition in new products and processes tomorrow. Thus IPRs and competition policy are now seen as complementary ways of achieving efficiency in a market economy. ${ }^{63}$

De igual manera, Nuno Pires de Carvalho ha establecido que el vínculo entre los derechos de patentes y la normativa de competencia se da en que los primeros promueven la creación y preservación de la diferenciación de los negocios, a través de la introducción al mercado de nuevos productos, lo que incrementa la competencia, logrando así una reducción de los costos transaccionales. ${ }^{64}$

[P]atent law and antitrust law are complementary legal tools that, from a macro-economic point of view, contribute to establish an efficient environment for the promotion of economic growth and wealth distribution. As explained above, intellectual property is about the protection of entrepreneurs differentials. And differentials only can be relevant in a scenario of rivalry (or the prospect thereof). Therefore, the role of enhancing and promoting the creation of differentials that intellectual property performs only can be achieved if rules that promote and preserve a competitive environment are in place and enforced. ${ }^{65}$

Por tanto, la teoría de que los derechos de patentes causan monopolización es el resultado de una mala comprensión del término monopolio, así como de una concepción distorsionada de la naturaleza exclusiva de los derechos de patentes. De ahí que el sistema de patentes no solo que no origina un monopolio de mercado, sino que es un instrumento legal que promueve la competencia, a través de la variedad de precios y productos, permitiendo así a los individuos a acceder a nuevos desarrollos en todas las áreas.

Consecuentemente, el plantear un régimen de protección para bienes que garantizan derechos fundamentales, en donde se permitan otros usos sin autorización del titular, más allá de las limitaciones y excepciones existentes, puede dar como resultado un debilitamiento del sistema de patentes existente, causando así un impacto negativo a la innovación, dados los costos sociales analizados anteriormente. Más aún cuando estas limitaciones y excepciones adicionales no solo recaerán sobre la inno-

63 Gallini Nancy T.; Trebilcock Michael J. "Intellectual Property Rights and Competition Policy: A Framework for the Analysis of Economic and Legal Issues", Robert D. Anderson and Nancy T. Gallini (eds.), Competition Policy and Intellectual Property in the Knowledge Based Economy, University of Calgary Press, Calgary, 1998.

64 Véase Pires de Carvalho, Nuno, The TRIPS Regime of Antitrust and Undisclosed Information 18-20, Kluwer Law International, The Netherlands, 2008.

65 Ibídem, p.41. 
vación protegida por el derecho de patentes, sino que "será aplicable también para los derechos que recaigan sobre la información no divulgada y los datos de prueba, sobre productos farmacéuticos y químico- agrícolas".

En el caso específico de la industria farmacéutica, este planteamiento del Código Ingenios, desconoce las características de la industria, los riesgos y los altos costos y tiempos de inversión. De esta manera, para ejemplificar la complejidad del proceso y sus altos costos, observemos los siguientes datos:

Desde que una compañía empieza a investigar una molécula hasta que la comercializa transcurren de media entre 10 y 15 años. De hecho, llegar a la fase de los ensayos clínicos, la más conocida para el ciudadano, supone unos cuatro años en los que hay que identificar un compuesto prometedor y hacerle las primeras pruebas, básicamente para comprobar su seguridad. Es la investigación básica o preclínica. Solo una de cada 10.000 moléculas estudiadas llega a convertirse en fármaco. [...] Con la autorización de las agencias reguladoras -FDA en Estados Unidos, MA en Europa, AEMPS en España- es cuando empiezan los ensayos clínicos. Otros seis o siete años. Hay un mantra que se repite en la industria: sacar adelante un medicamento cuesta 1.000 millones de dólares. Los datos más actualizados hablan de una inversión total de 1.172 millones de euros. ${ }^{66}$

Por tanto, dadas las características de la industria, sería poco probable que empresas farmacéuticas comercialicen y menos aún produzcan o innoven en el Ecuador, pues este régimen, sumado a la posible ampliación y extensión las limitaciones y excepciones establecidas en el ADPIC, no proporcionan los incentivos ni condiciones para recuperar la inversión. Lo que da como resultado, que las empresas farmacéuticas desarrolladoras de medicamentos migren de nuestro país por no tener las garantías suficientes para la protección de sus innovaciones, colocando así a los ciudadanos en una posición más vulnerable, pues en este escenario el acceso a medicamentos será prácticamente imposible.

Así, de aprobarse el código Ingenios con esta normativa, posiblemente nos estaríamos enfrentando a uno de los escenarios más críticos en lo relacionado con costos sociales, la imposibilidad de satisfacer las necesidades por la inexistencia de los productos.

\footnotetext{
66 Sevillano, Elena G. ¿Cuánto cuesta fabricar un medicamento? . El País, disponible en: http://economia.elpais.com/ economia/2015/03/20/actualidad/1426878852_197737.html
} 


\section{Conclusiones}

El Código Ingenios constituye una propuesta que va de la mano con la actual perspectiva del sistema de propiedad intelectual, buscar una propiedad intelectual más humana. Sin embargo, en este camino equivoca los medios, así con el objetivo de favorecer los intereses públicos, olvida la estructura y funcionamiento de los mercados, y del sistema de innovación, y promueve una política orientada a satisfacer las necesidades de los consumidores, mas no de los operadores económicos, que son quienes por medio de los inventores promueven la innovación tecnológica.

Un sistema de propiedad intelectual más humano, que satisfaga las necesidades y requerimientos de todos los actores, necesita de equilibrios. De esta manera, se necesita incentivos para inventores y operadores económicos, se precisa utilizar las flexibilidades del ADPIC de forma que se pueda explotar de mejor forma los resultados de la propiedad intelectual, se requiere de una normativa que utilice mecanismos creativos para promover la innovación. El sistema de propiedad intelectual por sí solo no traerá como resultado una mayor investigación y desarrollo, ni inversión extranjera, ni transferencia de tecnología. Se requieren de condiciones en el ambiente de mercado, político, económico y legal (seguridad jurídica). “|... (T)he significance of intellectual property rights in economic activity differs across countries and depends ( 1 ) on the amount of resources countries devote to creating intellectual assets as well as (2) the amount of protected knowledge and information used in production and consumption". ${ }^{67}$

El Código Ingenios no propone cambios significativos que mejoren las condiciones del actual sistema, los cambios sustantivos son más bien peligrosos porque, como hemos podido ver, hay un alto grado de posibilidades que su impacto afecte negativamente a la innovación. El resto de propuestas planteadas por el Código Ingenios, lejos de promover la innovación, pueden acarrear significativos obstáculos. No todo lo planteado es negativo, mas al analizar el proyecto en su totalidad representa un gran riesgo para la innovación, investigación y desarrollo. Además, del impacto a nivel internacional por implementar una normativa, que si bien promulga su consistencia con el ADPIC, posee fundamentos y bases distintas de éste, ya que puede generar inseguridad jurídica e inestabilidad en el mercado.

Por tanto, los esfuerzos deberían ser orientados a (1) promover la transferencia tecnológica, a través, de la formación del recurso humano, (2) generar incentivos económicos y no económicos a innovadores, la academia, emprendedores y operadores

67 Primo Braga, Carlos et. Al., Intellectual Property Rights and Economic Development 12, The World Bank, Washington D.C., 2000. 
económicos en general, (3) empoderar a los tenedores de conocimientos tradicionales como actores claves del proceso de innovación tecnológica y (4) desarrollar mecanismos legales creativos que permitan un mayor dinamismo del sistema de investigación. La tarea no es fácil, mas la retórica y los fundamentalismos no nos llevan a ningún lado. La promoción del conocimiento debe ser vista como una herramienta para garantizar los derechos fundamentales. No obstante, esto no puede realizarse a través de la limitación de derechos de propiedad intelectual, sino por medio de la activación de los sistemas de innovación, para la generación de más productos, a menor precio que permitan la satisfacción de necesidades y permitan una efectiva difusión del conocimiento, por medio de la divulgación y transferencia tecnológica. 


\section{Bibliografía}

Balick, Michael J., Traditional Knowledge: Lessons from the Past, Lessons for the Future, in Biodiversity and the Law, (Charles McManis ed., Earthscan, USA, 2007.

Biber-Klemm, S.; Cottier T, Right to Plant Genetic Resources And Traditional Knowledge Basic Issues and Perspectives, S.Biber-Klemm \& T. Cottier eds., UK, 2006.

Conde Gutiérrez Carlos Augusto, "Patentando ADN", Revista de la Propiedad Inmaterial No. 152011.

Correa Carlos M., Trade Related Aspects of Intellectual Property Rights, Oxford University Press, Great Britain, 2007.

Dutfield Graham, Protecting Traditional Knowledge: Pathways to the Future, International Centre for Trade and Sustainable Development (ICTSD), Switzerland, 2006

Gallini Nancy T.; Trebilcock Michael J. "Intellectual Property Rights and Competition Policy: A Framework for the Analysis of Economic and Legal Issues", Robert D. Anderson and Nancy T. Gallini (eds.), Competition Policy and Intellectual Property in the Knowledge Based Economy, University of Calgary Press, Calgary, 1998.

Lee Peter, "Patents, Paradigm Shifts, and Progress in Biomedical Science", The Yale Law Journal, Vol. 114, No. 3 (Dec., 2004), The Yale Law Journal Company, Inc., http://www.jstor.org/stable/4135694 . (acceso 20-04-2016).

Lewis Walter H. \& Ramani Veena, "Ethics and Practice in Ethnobiology: Analysis of the International Cooperative Biodiversity Group Project in Peru", Biodiversity and the Law, Charles McManis (ed.), Earthscan, USA, 2007.

Long Doris Estelle, "Traditional Knowledge and the Fight for the Public Domain", 5J.MARSHALL REV.INTELL.PROP.L., 317-321, 2006.

McManis, Charles, "Fitting Traditional Knowledge Protection and Biopiracy Claims into the Existing Intellectual Property and Unfair Competition Framework", Intellectual Property and Biological Resources, Burton Ong ed., Marshall Cavendish, Singapore, 2004.

Nuffield Council on Bioethics, The ethics of patenting DNA. A discussion paper, London, 2002. 
Pires de Carvalho Nuno, From the Shaman's Hut to the Patent Office: A Road Under Construction, in Biodiversity and the Law, (Charles McManis ed., Earthscan, USA, 2007).

The TRIPS Regime of Patent Rights (Nuno Pires de Carvalho ed., Kluwer Law ed. London, 2003).

_ Nuno, The TRIPS Regime of Antitrust and Undisclosed Information, Kluwer Law International, The Netherlands, 2008.

Posner, Richard A., "The Social Cost of Monopoly and Regulation", The Economics of Intellectual Property 3, Vol IV, Ruth Towse and Rudi Holzauer (eds.), Edward Elgar Publishing Limited, Cheltenham-UK, 2002.

Sevillano, Elena G.. ¿Cuánto cuesta fabricar un medicamento?. El País, disponible en: http://economia.elpais.com/economia/2015/03/20/actualidad/1426878852_197737.html. (acceso: 05-02-2016).

Straus, J., "Implications of the TRIPs Agreement in the field of patent law", From Gatt to TRIPs- The Agreement on Trade-Related Aspects of Intellectual Property Rights, F.K Beier and G. Schricker (eds.), Max Planck Institute for Foreign and International Patent, Munich, 1996.

Van Overwallem Geertrui, "Holder and User Perspectives in the Traditional Knowledge Debate: A European View", en Biodiversity and the Law, Charles McManis (ed.), Sterling, London, 2007.

Von Lewinski, Silke; Von Hahnm Anja, "Indigenous Heritage and Intellectual Property”, Silke von Lewinski \& Anja von Hahn (eds.), Kluwer Law International, The Netherlands, 2004.

WIPO, WIPO, Intellectual Property and Traditional Knowledge 6, WIPO Publication No. 920 (E), disponible en: http://www.wipo.int/edocs/pubdocs/en/tk/920/ wipo_pub_920.pdf (acceso: 21-04-2016).

WIPO/GRTKF/IC/13/7, of September 18, 2008- "Recognition of Traditional Knowledge within the Patent System".

\section{Legislación, jurisprudencia y directrices}

35 United States Code.

Acuerdo sobre los aspectos de los Derechos de Propiedad Intelectual relaciona- 
dos con el Comercio, disponible en https://www.wto.org/spanish/docs_s/ legal_s/27-trips_02_s.htm (acceso:12-04-2016).

Código Orgánico de Economía Social del Conocimiento e Innovación, disponible en http://coesc.educacionsuperior.gob.ec/index.php/C\%C3\%B3digo_ Org\%C3\%Alnico_de_Econom\%C3\%ADa_Social_del_Conocimiento_e_ Innovaci\%C3\%B3n (acceso: 11-03-2016).

Constitución de la República del Ecuador. Registro Oficial No.449 de 20 de octubre de 2008.

Directiva 98/44/CE del Parlamento Europeo y del Consejo relativa a la protección jurídica de las invenciones biotecnológicas. Artículo 3.2 de 6 de julio de 1998.

Ethicon, Inc. vs United States Surgical Corporation, 937 F.Supp. 1015 (United States District Court, D. Connecticut, 9 September 1996).

International Conference on Intellectual Property and Development, Ginebra 7-8 Abril 2016.

Monsanto Co. vs Kamp, 269 F.Supp.818, District of Columbia, District Court, 15 June 1967.

Shields vs Halliburton Co., 667 F.2d 1232 (US Court of Appeals, 5th Circuit, 19 February 1982).

United States Supreme Court. Association for Molecular Pathology et al., Petitioners v .Myriad Genetics, Inc., et al., No. 12-398, 13 de junio de 2013. 2013 WL 2631062.

United States Supreme Court. Diamond v. Chakrabarty, 447 U.S. 303, 1980. 